\title{
Le: \\ MODELAGEM DO FLUXO DAS ÁGUAS SUBTERRÂNEAS EM ÁREA DE CONSERVAÇÃO NO SUDOESTE PAULISTA
} BIOENG

\author{
M. P. M. A. Pinheiro ${ }^{{ }^{*}}$, R. L. Manzione ${ }^{2}$
}

${ }^{1}$ UNESP - Universidade Estadual Paulista, Faculdade de Ciências Agronômicas, Campus de Botucatu, SP, Brasil

${ }^{2}$ UNESP - Universidade Estadual Paulista, Faculdade de Ciências e Engenharia, Campus de Tupã, SP, Brasil

Article history: Received 13 June 2019; Received in revised form 15 August 2019; Accepted 23 August 2019; Available online 30 September 2019.

\section{RESUMO}

Este trabalho foi desenvolvido para fornecer um conhecimento específico sobre o comportamento hidrogeológico da área da Estação Ecológica de Avaré (EEcAv), especialmente sobre a dinâmica do fluxo da água subterrânea, mostrando a importância da preservação das áreas de conservação ambiental do Estado de São Paulo para segurança hídirca. A área de estudo, está localizada no município de Avaré/SP, ocupa uma extensão territorial de aproximadamente 720 hectares. Para este estudo, foram utilizados: um modelo digital de terreno SRTM (Shuttle Radar Topography Mission) com resolução de 30 metros; e os seguintes softwares, para subsidiar a análise: QGIS, SAGA e MapWindow (extensão TauDEM). Com a ajuda dessas ferramentas, foi possível modelar o fluxo de água subterrânea estimando a Direção do Fluxo, Fluxo Acumulado e Índice de Umidade Topográfica (ITU) da área. Com os resultados obtidos, verificou-se que o fluxo subterrâneo da EEcAv está direcionado para os locais com maior umidade, ou seja, os rios Novo e Pardo; e também mostra que a estação está situada entre corredores hídricos reforçando sua importância ambiental.

Palavras-chave: mapeamento de fluxo subterrâneo, unidade de conservação, sensoriamento remoto.

\section{GROUNDWATER FLOW MODELING IN CONSERVATION AREA IN SOUTHWEST PART OF SÃO PAULO STATE, BRAZIL}

\begin{abstract}
This work was developed to provide a specific knowledge about the hydrogeological behavior of the Avaré Ecological Station (EEcAv) area, especially the groundwater flow dynamics, showing the importance of preserving the environmental conservation areas of the State of São Paulo for water security. The study area located in the municipality of Avaré/SP-Brazil occupies a territorial extension of approximately 720 hectares. For this study a 30-meter Shuttle Radar Topography Mission (SRTM) digital elevation model was used; and the following software to support the analysis: QGIS, SAGA and MapWindow (extension TauDEM). With the help of these tools it was possible to model groundwater flow by estimating the Flow Direction, Accumulated Flow and Topographic Moisture Index (ITU) of the area. With the obtained results it was verified that the underground flow of the EEcAv is
\end{abstract}

medeirosmirian@yahoo.com.br 
directed to the places with higher humidity, that is, the Novo and Pardo rivers. It also shows that the station is situated between water corridors reinforcing its environmental importance.

Keywords: groundwater flow mapping, conservation unit, remote sensing.

\section{INTRODUÇÃO}

É de conhecimento mundial que, o Brasil é um país com complexos e diversos biomas, assim como uma extensão territorial significativa, compondo assim um cenário desafiante para a obtenção de informações e o uso do espaço geográfico nacional. Nesse contexto, as geotecnologias são um dos instrumentos que auxiliam na gestão e monitoramento territorial (EMBRAPA, 2013). A junção de tecnologias para coleta, processamento, análise e oferta de informações com referência geográfica, é conhecida com geotecnologia; a mesma pode ser dividida em 3 partes: hardware, software e peopleware que, unidas são excelentes instrumentos que têm como objetivo final auxiliar na tomada de decisão (ROSA, 2005).

A utilização de dados geoespaciais pode favorecer, praticamente, todo planejamento e gerenciamento de atividades agrícolas, em âmbito não somente nacional, como regional e local, exemplos funcionais disso, são os usos de imagens de satélite e mapas digitais. Do ponto de vista ecológico, o uso dessas ferramentas possibilitaria a obtenção de informações mais precisas da real condição do local analisado. A sustentação/conservação dos processos ecológicos em áreas protegidas é imprescindível, visto que, a previsão para os próximos 40 anos é uma redução da biodiversidade de 15 a $37 \%$ das espécies vegetais (THOMAS et al., 2004; PEREIRA et al., 2010).

Pela lei (9.985/2000), as Unidades de Conservação (UCs) são basicamente "espaços territoriais, juntamente, com todos os seus recursos naturais, protegidos e preservados pelo Poder Público". Segundo o MMA (2019), as UCs são divididas em duas classes: Proteção
Integral e Uso Sustentável. A diferença entre elas é a permissão da realização de atividades exploratórias diretas ou indiretas dos recursos naturais, ou seja, se é permitido ou não o consumo, coleta ou dano ao meio ambiente local. Estação Ecológica é uma das cincos categorias pertencentes à Proteção Integral, juntamente com Reserva Biológica, Parque Nacional, Monumento Natural e Refúgio da Vida Silvestre, e diferentemente das demais categorias é uma área cuja visitação é permitida somente com objetivos educacionais, e que possui como funções preservar os recursos naturais e realizar pesquisas científicas.

A Estação Ecológica de Avaré (EEcAv) que foi criada em 2010, originalmente, com o nome de Horto Florestal Andrada e Silva, como uma unidade de conservação de proteção integral estadual, por possuir amostras dos biomas Cerrado (em sua maioria) e Mata Atlântica. Ambos altamente importantes por suas biodiversidades, pelo alto grau de endemismo e por estarem entre os mais ameaçados do planeta (MYERS et al., 2000; MITTERMEIER, 2005).

Há uma ligação direta entre o tipo de vegetação, a conservação do solo e a interação entre águas subterrâneas e águas superficiais, um depende do outro (MANZIONE, 2015). Quando há um desequilíbrio todo o ciclo é afetado. No caso de áreas verdes, por exemplo, estações ecológicas, um desbalanceamento poderia afetar a qualidade das águas (como a presença de compostos químicos presentes em agroquímicos) superficiais e subterrâneas alterando a água utilizada para irrigação nas propriedades circunvizinhas, podendo gerar modificações na qualidade do produto final. Do ponto de vista hidrológico, essas 
áreas possuem um papel importante no fornecimento de água, recarga de aquíferos e amortecimento do escoamento superficial.

Sendo assim, o objetivo desse trabalho foi mapear o fluxo das águas

\section{MATERIAL E MÉTODOS}

\section{Área de Estudo}

A área de estudo é a Estação Ecológica de Avaré (EEcAv), antigo Horto Florestal Andrada e Silva, localizada no subterrâneas presentes na Estação Ecológica de Avaré (EEcAv), evidenciando a importância da estação no cenário hidrológico local.

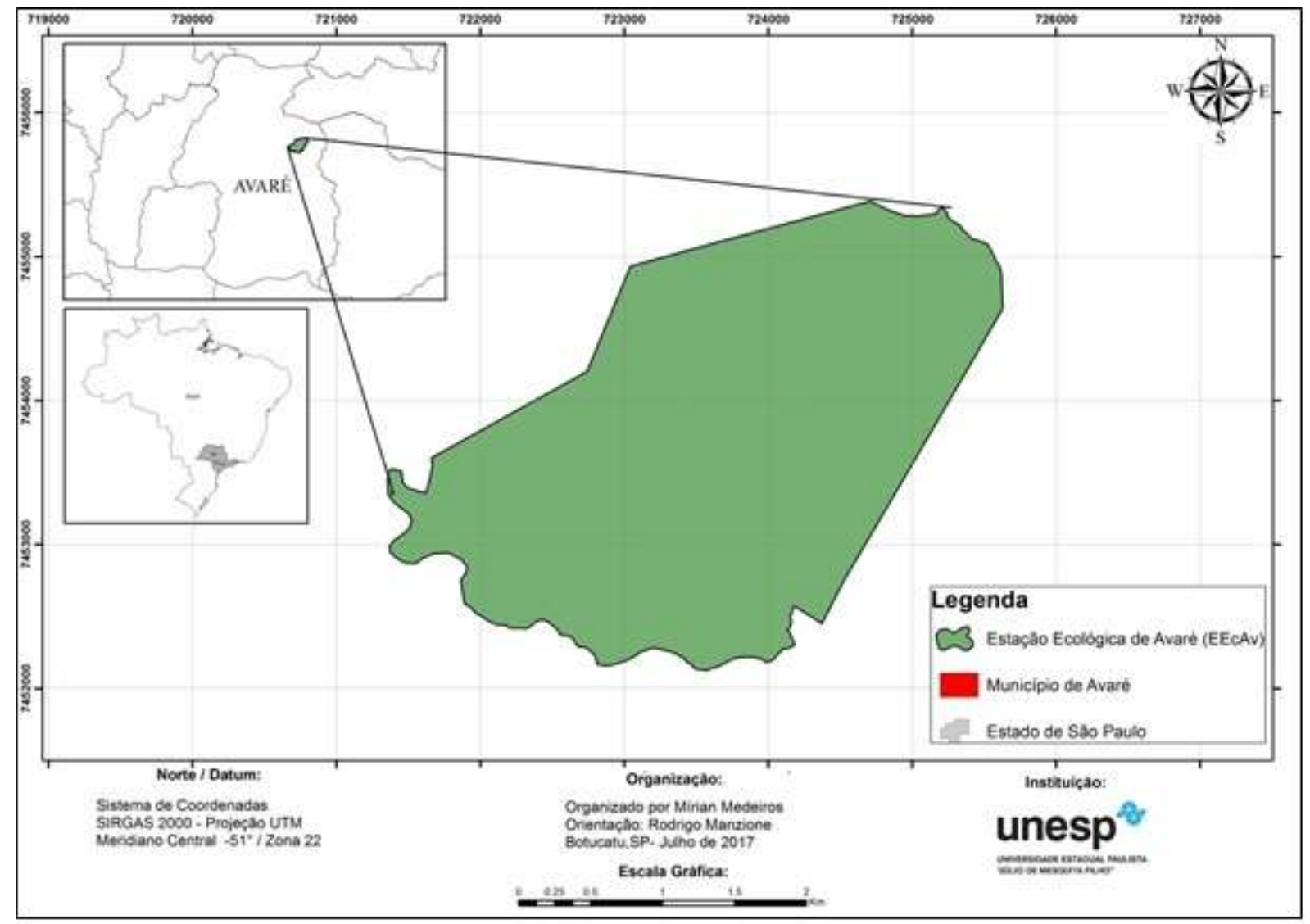

Figura 1. Localização da área de estudo, município de Avaré, SP.

De acordo com o Mapa Geomorfológico do IPT (1981), a área estudada (EEcAv) está localizada sob a Formação Marília do Grupo Bauru, formando junto com as Formações Adamantina, Santo Anastácio e Caiuá, o Sistema Aquífero Bauru (SAB). Nessa formação podem estar presentes arenitos de granulação fina a grossa, compreendendo bancos maciços com tênues estratificações cruzadas de médio porte, podendo incluir lentes e município de Avaré/SP, que está situado na região sudoeste do Brasil (Figura 1), ocupa uma extensão territorial de, aproximadamente, 720 hectares. 
Sistema Aquífero Serra Geral podem intercomunicar as camadas superiores do SAB com outro importante sistema aquífero, o Sistema Aquífero Guarani (SAG).

\section{Caracterização Física da Área de Estudo}

Para elaboração dos mapas temáticos foram utilizados os seguintes programas: QGIS (versão 2.8.3); MapWindow GIS (versão 4.8.8), com extensão do pacote TauDEM e SAGA (versão 2.1.2). Foram trabalhados três materiais sobre $\mathrm{O}$ mapeamento do fluxo das águas subterrâneas: o mapa de Direção de Fluxo, o mapa de Fluxo Acumulado e o Índice Topográfico de Umidade (ITU).

\section{Direção e fluxo acumulado}

Para a elaboração dos mapas de Direção de Fluxo e Fluxo Acumulado, a metodologia foi dividida em três etapas: (1) construção do Modelo Digital do Terreno (MDT) com níveis freáticos, (2) eliminação das depressões artificiais e (3) cálculo da direção do fluxo.

Etapa 1: Na construção do MDT com níveis freáticos, foram utilizados dados da missão SRTM (Shuttle Radar Topography Mission) disponível em https://earthdata.nasa.gov/, com resolução de 30 metros e os dados piezométricos de seis poços coletados manualmente na EEcAv com um medidor de nível, tipo fita (Figura 2). Os dados dos poços foram convertidos em uma camada vetorial, essa camada foi interpolada ao MDT pelo método IQD (Inverso do Quadrado da Distância) por não haver dados suficientes para mensurar a dependência espacial das amostras por métodos geoestatísticos (YAMAMOTO \& LANDIM, 2013). A partir da álgebra de mapas, houve uma subtração do MDT pela profundidade dos níveis freáticos gerando assim uma superfície que representa a cota dos níveis freáticos. Esse processo foi realizado no programa QGIS e repetido para cada período coletado. As coletas de dados dos piezômetros presentes nos poços (Tabela 1) foram realizadas em duas datas, representando momentos de menor $(25 / 06 / 2014)$ e maior (11/12/2017) fluxo devido às estações do ano, inverno e verão, respectivamente. Posterior ao aprofundamento da drenagem, com a finalidade de conservar a conectividade do fluxo ao longo do canal, realizou-se o preenchimento das depressões artificiais e o cálculo da direção de fluxo.

Etapa 2: $\mathrm{O}$ arquivo gerado pela Etapa 1 foi trabalhado no programa MapWindow GIS com uma extensão do pacote TauDEM, nessa etapa foram identificadas e eliminadas a ocorrência de imperfeições (depressões artificiais ou espúrias) que foram preenchidas de forma a possibilitar uma continuidade do fluxo. As imperfeições podem ter duas causas: erros introduzidos durante $\mathrm{o}$ processo de interpolação ou erros existentes nos dados de entrada (TRIBE, 1992; GARCIA; CAMARASA, 1999).

Segundo Oliveira et al. (2007a), as imperfeições são normalmente centralizadas em ambientes com alta umidade, como locais de inundação, ou próximo a determinados tipos de paisagem onde um MDT, com uma resolução baixa, não consiga representá-los, como depósitos de sedimentos ou dunas. São diversos os métodos propostos para o preenchimento das imperfeições. Jenson; Domingue (1988) desenvolveram o método mais seguro, que apenas eleva as células contidas dentro das imperfeições. $\mathrm{O}$ algoritmo desenvolvido por eles possui dois pontos gerais: (a) atribuir direções de fluxo de acordo com a maior declividade (b) adicionar de forma artificial uma altitude às células ou conjunto de células que compõem as áreas de depressão, até que seja obtido a direção do escoamento (BUARQUE et al., 2009).

Etapa 3: Ainda trabalhando no MapWindow GIS, iniciou-se o procedimento de determinação das direções do fluxo, o método escolhido foi o D8. Segundo Oliveira et al. (2007b), o método D8 é a metodologia mais usual para a delimitação da estrutura de drenagem. O cálculo desse método é 
baseado na determinação da direção do fluxo de cada célula da grade sendo considerada a maior declividade entre a célula central e as oito células circunvizinhas (N, NE, L, SE, S, SO, O e NO) (Figura 3), sendo que o escoamento sempre ocorre para uma das oitos células (O’CALLAGHAN; MARK, 1984).

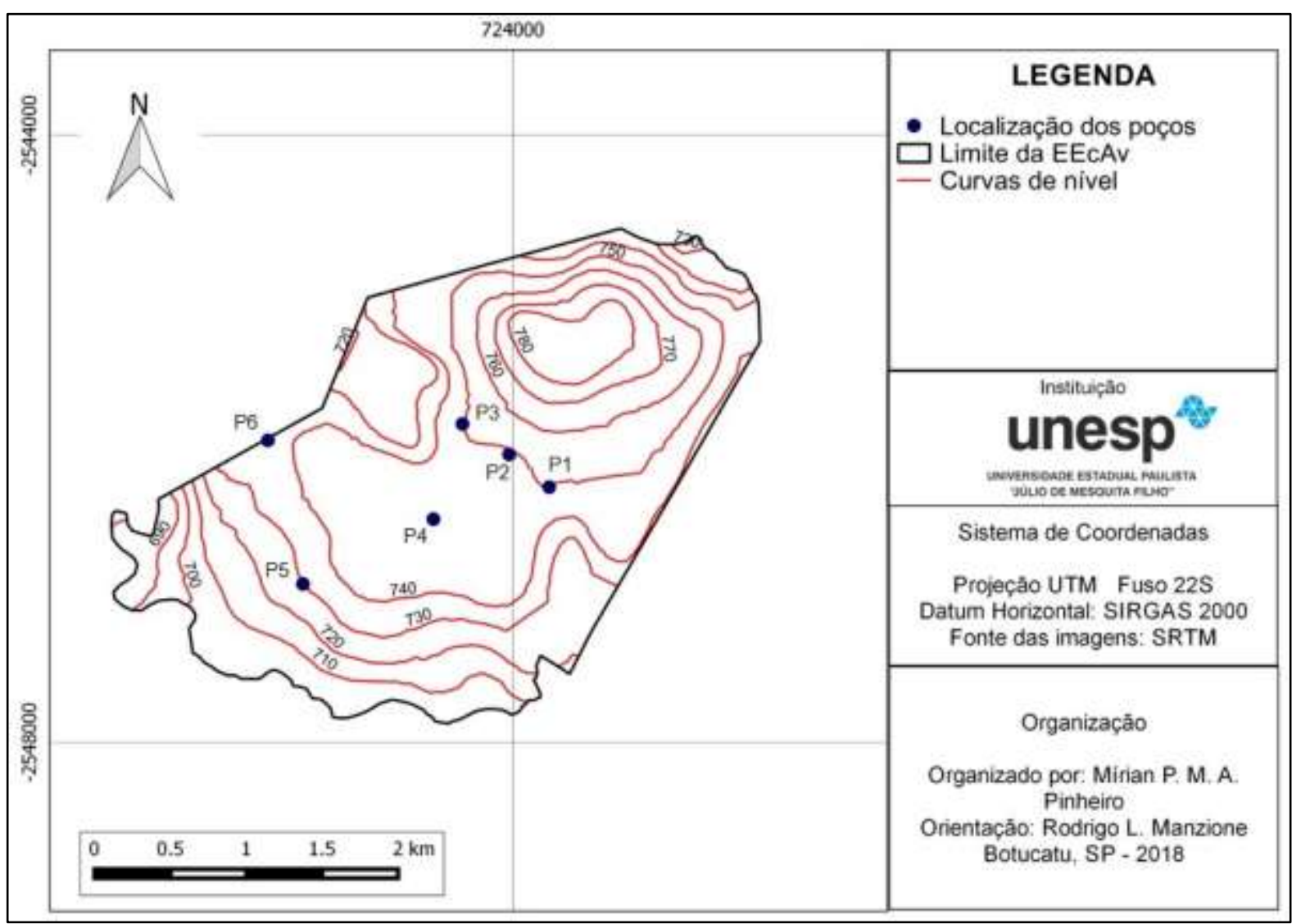

Figura 2. Distribuição dos poços na EEcAv.

Tabela 1. Dados dos piezômetros referente aos níveis de água nos seis poços

\section{Períodos}

\begin{tabular}{lcc} 
& $\mathbf{2 5 / 0 6 / 2 0 1 4}$ & $\mathbf{1 1 / 1 2 / 2 0 1 7}$ \\
\hline Poço 1 & $13,95 \mathrm{~m}$ & $11,40 \mathrm{~m}$ \\
Poço 2 & $18,78 \mathrm{~m}$ & $15,53 \mathrm{~m}$ \\
Poço 3 & $15,26 \mathrm{~m}$ & $12,43 \mathrm{~m}$ \\
Poço 4 & $16,52 \mathrm{~m}$ & $12,92 \mathrm{~m}$ \\
Poço 5 & $12,85 \mathrm{~m}$ & $10,51 \mathrm{~m}$ \\
Poço 6 & $8,50 \mathrm{~m}$ & $7,07 \mathrm{~m}$ \\
\hline
\end{tabular}




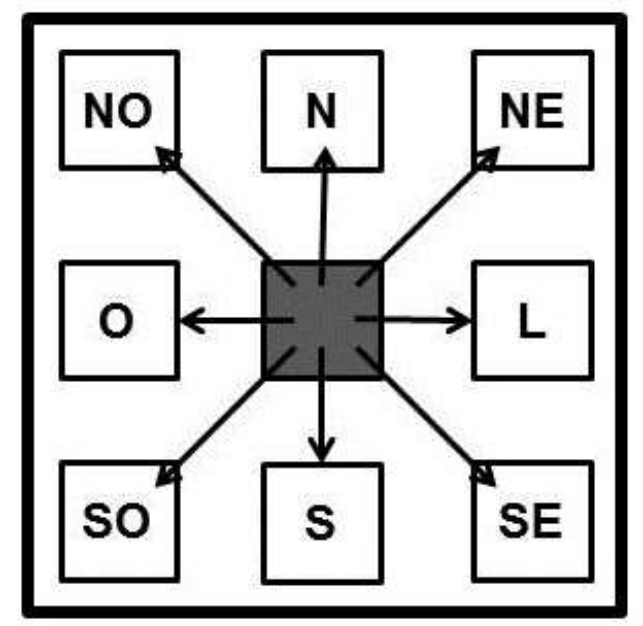

Figura 3. Codificação utilizada para distinguir as oito direções de fluxo possíveis no método D8.

Para finalização dos mapas de Direção de Fluxo, os arquivos gerados foram novamente trabalhados no QGIS para evidenciar o comportamento da direção do fluxo para cada período coletado.

De acordo com Valeriano (2007), fluxo acumulado também conhecido como área de captação, é um parâmetro que mostra o nível de afluência do escoamento, sua obtenção, seja ela manual ou computacional, é complexa, pois unifica características de comprimento de rampa (conexão com divisores de água a montante) e a curvatura horizontal (afluência e divergência das linhas de fluxo). O mapa de Fluxo Acumulado foi concluído após as 3 etapas citadas acima, esse é um mapa único, pois o fluxo é contínuo, independente das coletas dos níveis freáticos.

\section{Índice Topográfico de Umidade (ITU)}

Quanto ao mapa do ITU, a metodologia aplicada é diferente dos demais mapas elaborados nos procedimentos anteriores. Para confecção desse mapa, usando o MDT como base, foi utilizado o programa SAGA, e dividido em duas etapas: (1) área de contribuição e declividade, e (2) cálculo do ITU.

Etapa 1: Elaborar um mapa de fluxo da área de contribuição, utilizando o método D-infinity que, diferentemente, do método D8 atribui múltiplas direções para o fluxo. Em seguida é elaborado o mapa de declividade.

O cálculo D8 apresenta-se apropriado para zonas de fluxo convergente e para vales definidos, no entanto, para demais situações erros são verificados. De acordo com Tarboton (1997); Costa-Cabral; Burges (1994) e Quinn et al. (1991), outros métodos são sugeridos considerando uma direção de fluxo não discreta ou um fluxo em múltiplas direções. Quando comparado com o método D8, os métodos que utilizam fluxos não discretos apresentam uma diminuição nos erros relativos à direção de fluxo, tendo como consequência, uma melhor delimitação do canal (FREEMAN, 1991; DESMET; GOVERS, 1996, RAMOS et al., 2003).

Etapa 2: Após a obtenção dos mapas na Etapa 1, e utilizando a álgebra de mapas é confeccionado o mapa de ITU, através do Log natural $(\ln )$, da divisão do mapa da área de contribuição (Ac) específica por unidade de largura ortogonal à linha de fluxo $\left(\mathrm{m}^{-2} \mathrm{~m}^{-1}\right)$, que é uma medida que simula o volume potencial do escoamento superficial num determinado ponto da vertente, pela tangente do mapa de declividade local (tg $\beta$ ) (Equação 1) (BEVEN; KIRKBY, 1979, WILSON; GALLANT, 2000). 


$$
\mathrm{ITU}=\ln \left(\frac{\mathrm{Ac}}{\operatorname{tg} \beta}\right)
$$

O ITU é um índice topográfico secundário, pois foi calculado a partir da combinação de dois índices primários (a área de contribuição e a declividade da área). E sua principal utilização é para identificar as zonas de saturação

\section{RESULTADOS E DISCUSSÃO}

Para facilitar uma melhor compreensão do comportamento do fluxo da área, foi desenvolvido, a partir do SRTM e através da drenagem numérica, os mapas da hidrografia (Figura 4) e declividade (clinográfico) (Figura 5) da área de estudo.
(Equação 1)

superficial, evidenciando na área os pontos de acúmulo de água, ou seja, é um indicativo da disponibilidade, em longo prazo, da umidade do solo em um certo ponto da paisagem (MOORE, et al. 1993).

\begin{abstract}
Observando as declividades apresentadas na Figura 5, é possível afirmar que a maioria da área da EEcAv apresenta declive plano e suave ondulado e não possui os relevos montanhoso $\mathrm{e}$ escarpado. De acordo com os seis intervalos distintos sugeridos pela EMBRAPA (1979) (Tabela 2).
\end{abstract}

Tabela 2. Classificação da declividade, de acordo com EMBRAPA (1979)

\begin{tabular}{|c|c|}
\hline Declividade & Descrição \\
\hline 0 a $3 \%$ & Relevo plano \\
\hline 3 a $8 \%$ & Relevo suave ondulado \\
\hline 8 a $20 \%$ & Relevo ondulado \\
\hline 20 a $45 \%$ & Relevo forte ondulado \\
\hline 45 a $75 \%$ & Relevo montanhoso \\
\hline$>75 \%$ & Relevo escarpado \\
\hline
\end{tabular}




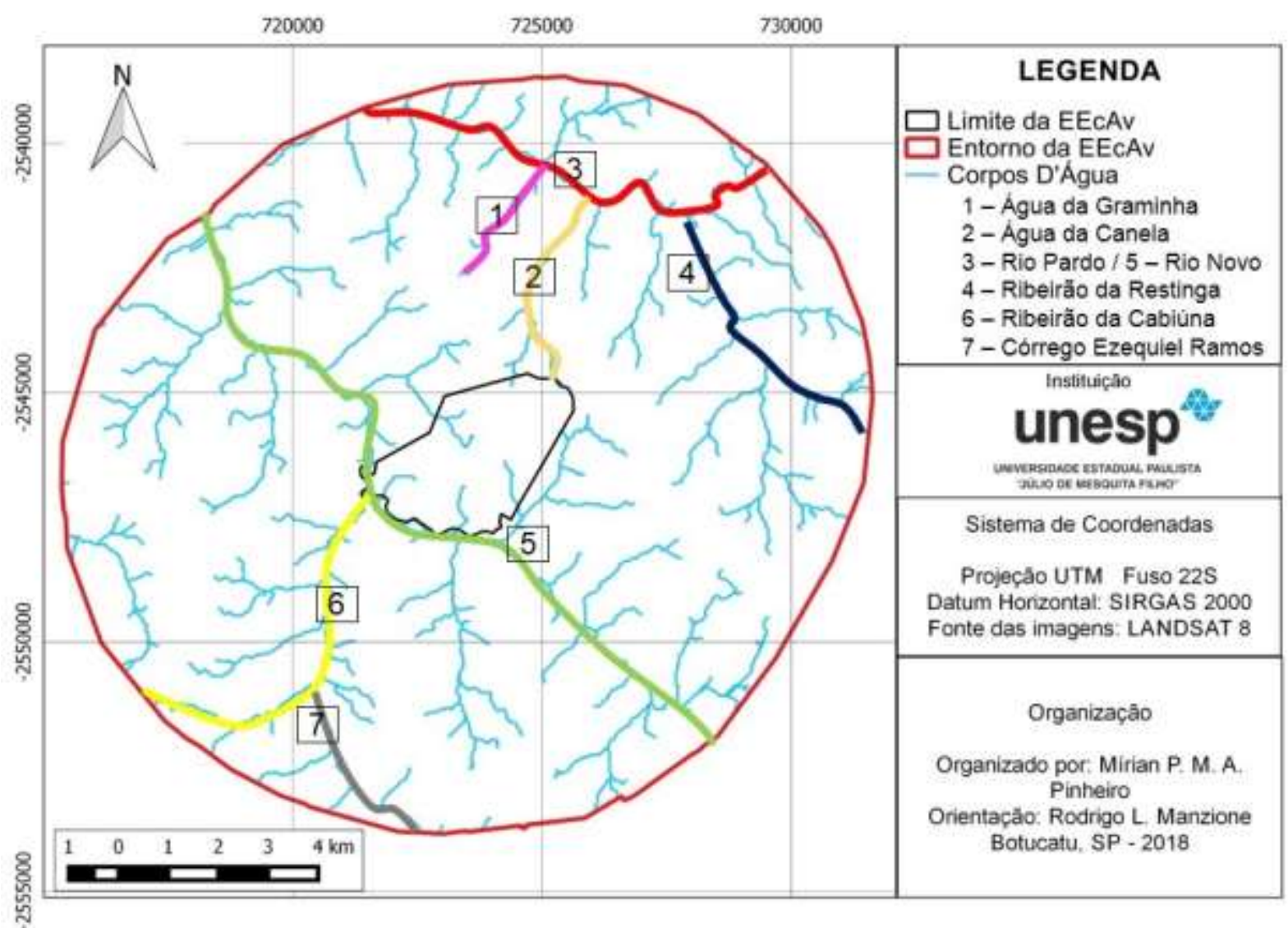

Figura 4. Hidrografia da EEcAv e seu entorno.

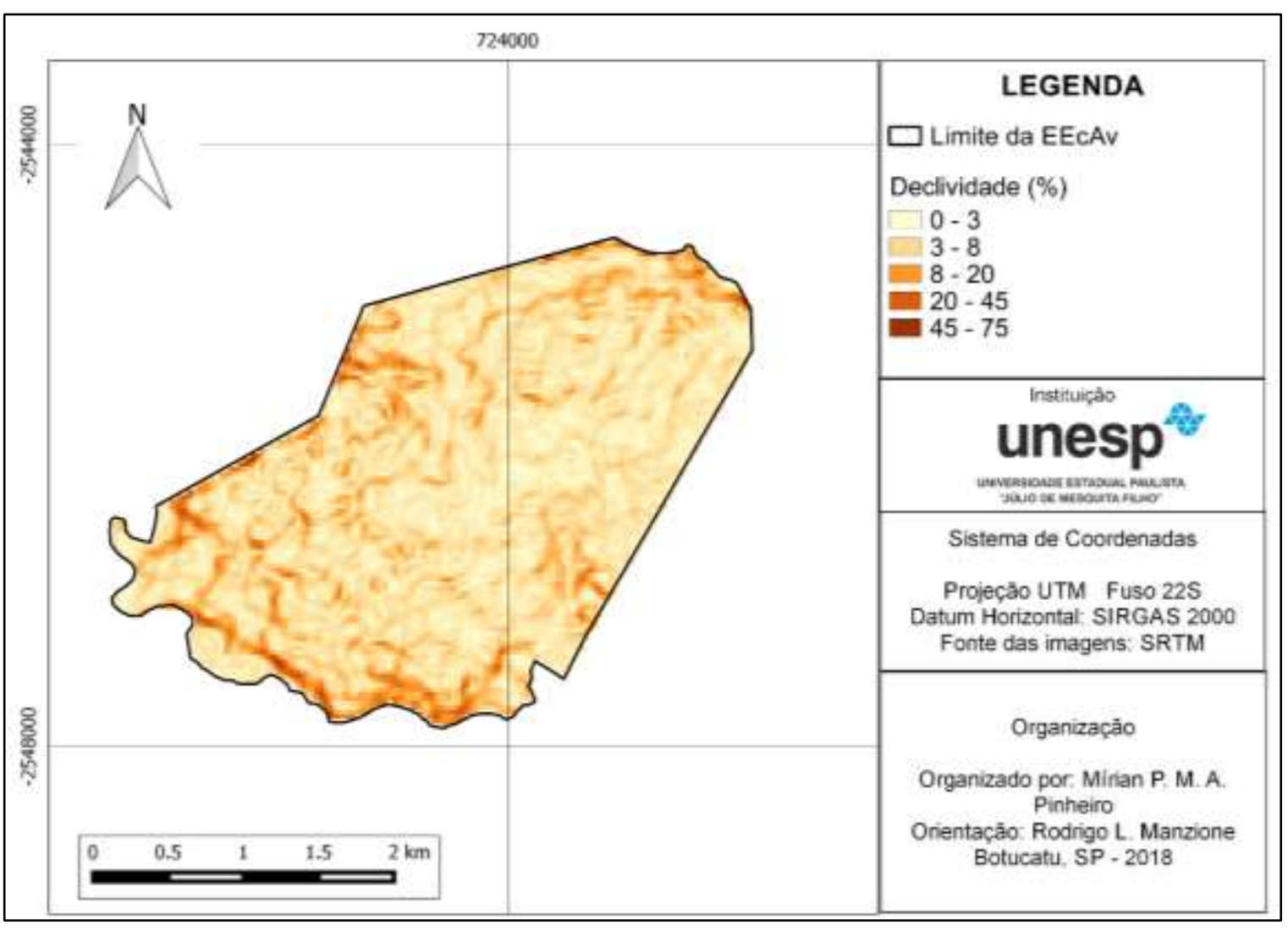

Figura 5. Declividade (mapa clinográfico) elaborado a partir do SRTM. 


\section{Modelagem e Mapeamento do Fluxo das Águas Subterrâneas}

\section{Direção de Fluxo}

A área da EEcAv é circundada pelo Córrego Água da Canela e pelo Rio Pardo. Analisando os dois mapas de direção de fluxo para as datas propostas, é perceptível que não houve alterações na direção do fluxo subterrâneo durante os períodos. Também pode ser observado comportamentos bem semelhantes, nas regiões norte e sul da área de estudo. Ao norte, é possível perceber que há uma distribuição do fluxo, tanto para fora quanto da dentro da EEcAv. Ao sul, que o fluxo é direcionado para onde está localizado o Rio Novo. Na parte norte da estação encontram-se as elevações mais altas, com aproximadamente 803 metros acima do nível do mar, que é considerado o divisor de águas principal dentro da área de estudo. Usando essa elevação como ponto de referência, o fluxo flui em todas as direções, o fluxo que segue para o norte deságua no pelo Córrego Água da Canela. Já o fluxo que segue para o sul é distribuído seguindo a declividade da área e parte dessa distribuição deságua no Rio Novo que corre ao longo de toda a região sul da EEcAv. As Figuras 6 e 7 exibem os mapas correspondente as direções do fluxo para cada ambos períodos.

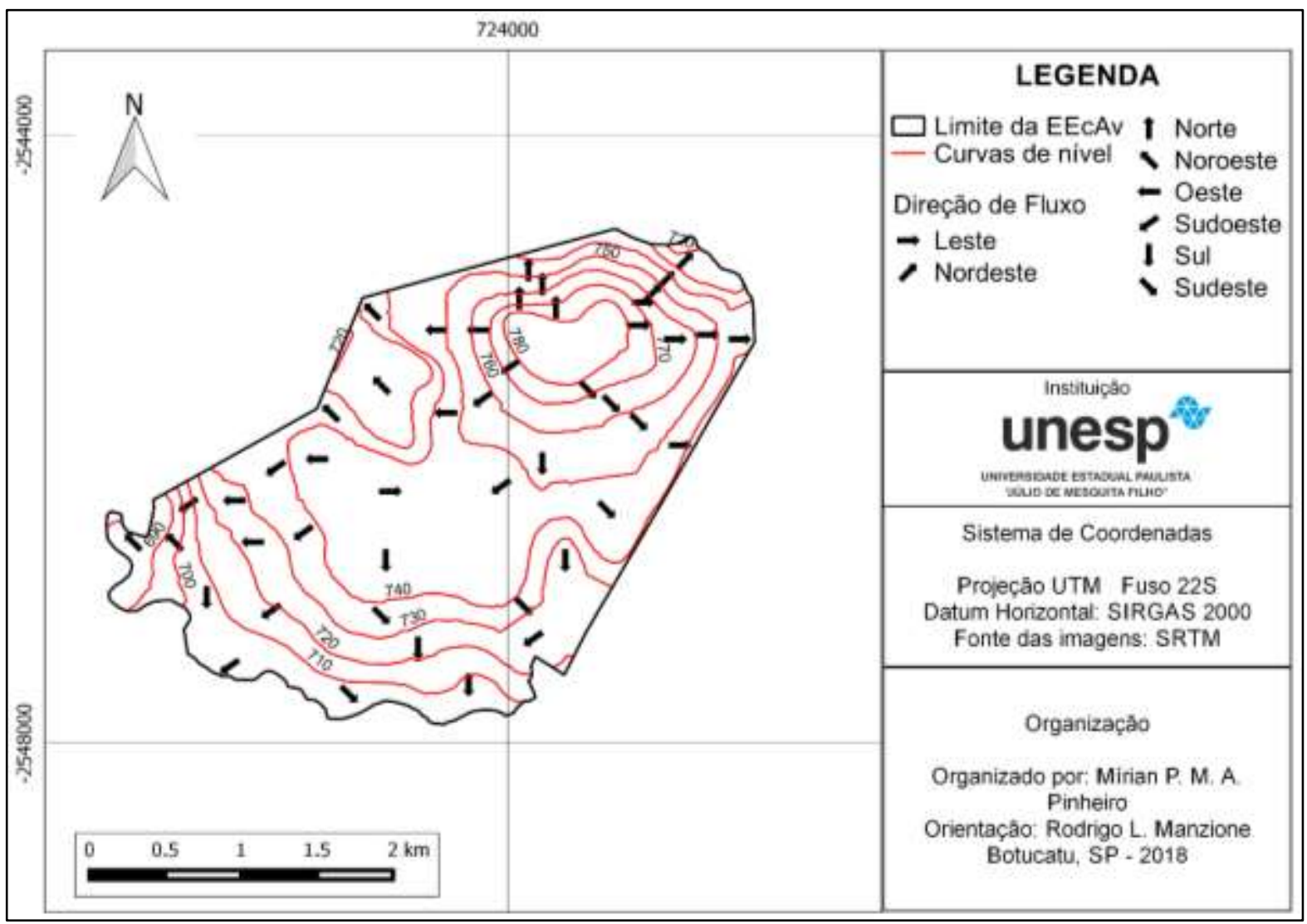

Figura 6. Direção do fluxo no dia 25/06/2014. 


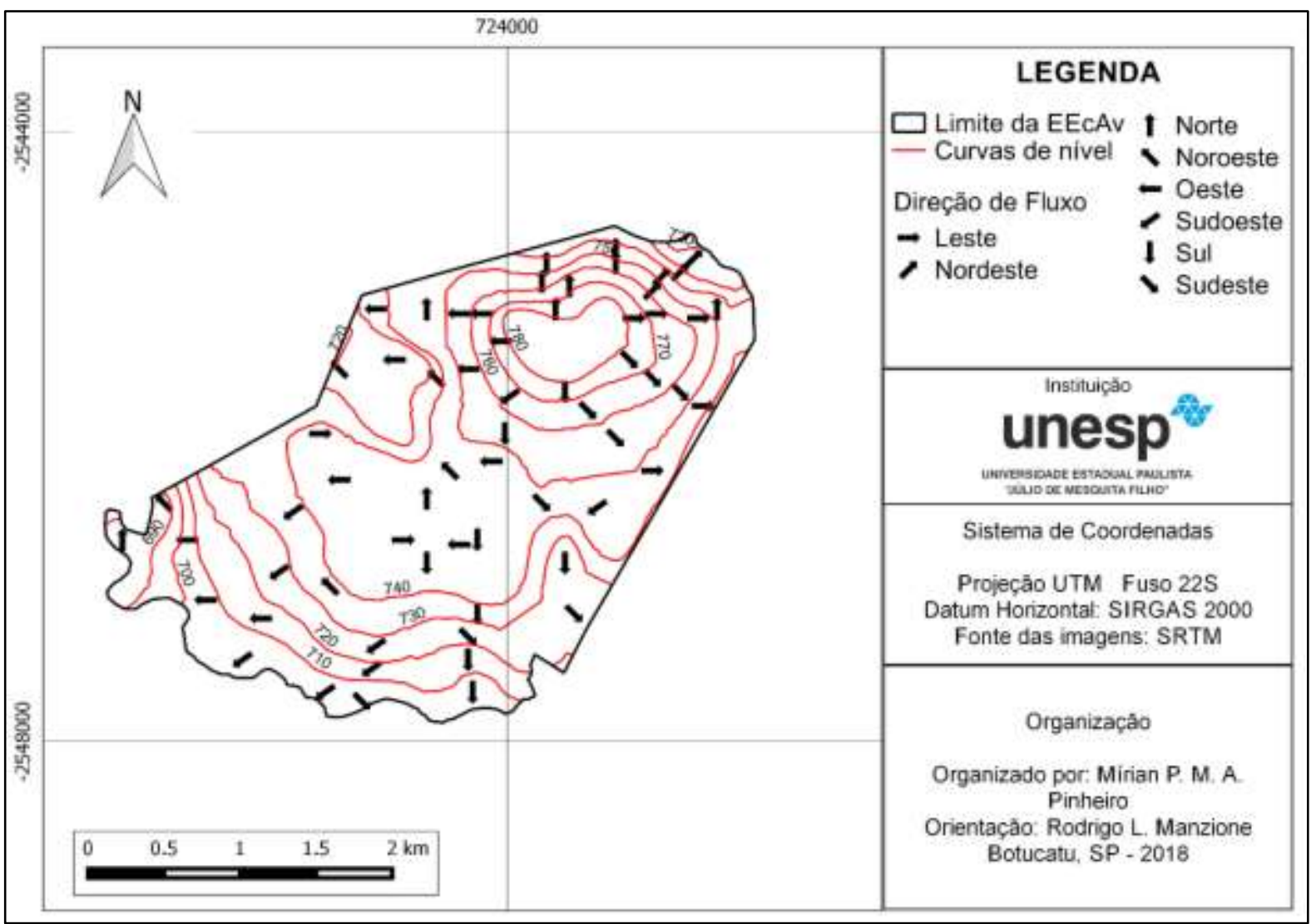

Figura 7. Direção do fluxo no dia 11/12/2017.

Santarosa (2016) trabalhando com estimativa da direção dos fluxos subterrâneos na Estação Ecológica de Santa Bárbara (EEcSB), notou alterações nos níveis piezométricos entre períodos

\section{Fluxo Acumulado}

O fluxo acumulado é obtido através da somatória da área (quantidade) de células presente na área de escoamento (direção do fluxo) (SOBRINHO et al., 2010). Segundo Tucci (1997), a área de captação é considerada uma área de agrupamento hídrico natural das chuvas, na qual o fluxo do escoamento superficial é direcionado a um único ponto de saída, o exutório. Assim, uma sub-bacia é composta pela rede de drenagem do rio principal e por várias superfícies (vertentes) (MELLEK, 2012). Após a distintos. Safre (2018) também trabalhando com níveis potenciométricos na $\mathrm{EEcSB}$ observou um comportamento semelhante do aquífero para sua área de estudo.

elaboração do mapa de direção do fluxo, foi elaborado o mapa com o fluxo acumulado, que indica os locais preferenciais onde o fluxo de concentra. Na Figura 8 é possível observar que, as linhas com cores mais escuras são aquelas com maiores concentrações de fluxo (os rios), sendo assim o fluxo acumulado presente na área selecionada (área de estudo e seu entorno) está direcionado para o Rio Novo, ao sul (parte inferior do mapa), e para o Rio Pardo, ao norte (parte superior do mapa). 


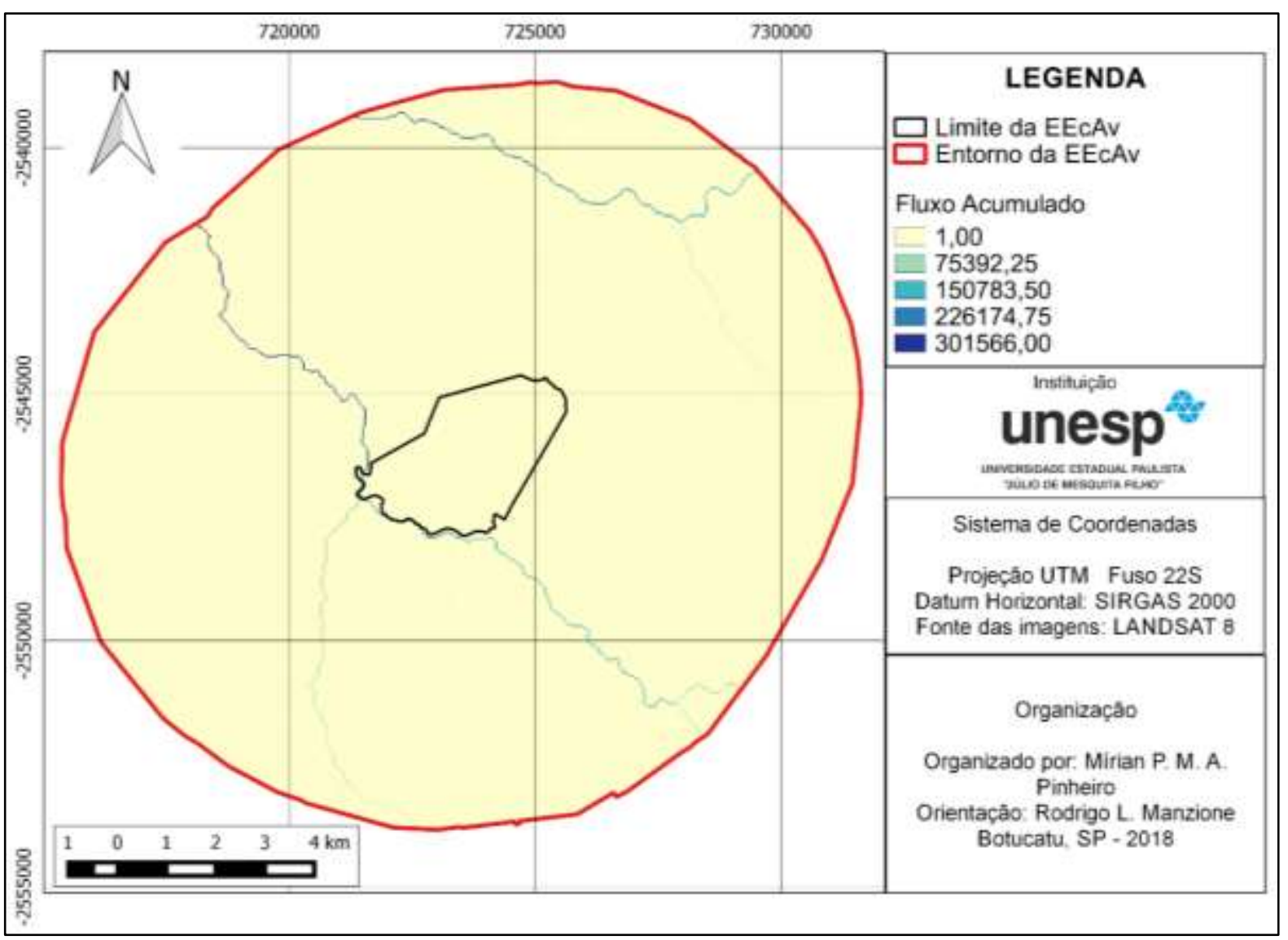

Figura 8. Fluxo Acumulado da EEcAv e seu entorno

\section{Índice Topográfico de Umidade (ITU)}

A Figura 9 apresenta o mapa do ITU da área de estudo e seu entorno. Podemos observar um comportamento bem parecido com o mapa de Fluxo Acumulado. Na figura percebemos que os valores variam entre 7 e 26 , sendo 7 o local com menor umidade e 26 o local com maior umidade. As linhas azuis mais espessas ao norte e ao sul do mapa, são os rios Pardo e Novo. Capoane et al. (2015) trabalhando com ITU numa bacia hidrográfica no RS, constatou que um MDT gerado a partir de carta topográfica apresenta resultados inadequados para indicar áreas com maior probabilidade de saturação hídrica no solo, pois possui limitações de precisão posicional e vertical. Oliveira et al. (2012) observaram que o índice topográfico de umidade e a declividade foram características do relevo que obtiveram maior correlação com outros atributos avaliados do solo, um exemplo é o teor de matéria orgânica. De acordo com Gallant e Wilson (2000), a velocidade do fluxo superficial da água, o teor de água no solo e o potencial de erodibilidade são afetada diretamente pela declividade. Além do relevo, outras variáveis possuem influência direta sobre o comportamento dos níveis freáticos, como Nava e Manzione (2015) demonstram os efeitos da precipitação e da evapotranspiração na resposta das águas subterrâneas à estímulos climatológicos. Santarosa \& Manzione (2018), utilizando uma rede de monitoramento de águas subterrâneas com um número de poços superior a 50 conseguiram incorporar informações secundárias de terreno aos dados de poços no processo de interpolação, resultando em um mapa com erros menores do que aqueles esimados somente utilizando dados de nível dos poços. Resultados como os gerados nesse estudo podem complementar bases de dados sobre variáveis hidrológicas e auxiliar nos processos de tomada de decisão em recursos hídricos quantificando a incerteza associada a previsão e incorporando-a aos instrumentos de gestão de recursos hídricos, como planos, outorga, 
enquadramento e até mesmo a cobrança

(MANZIONE, 2014).

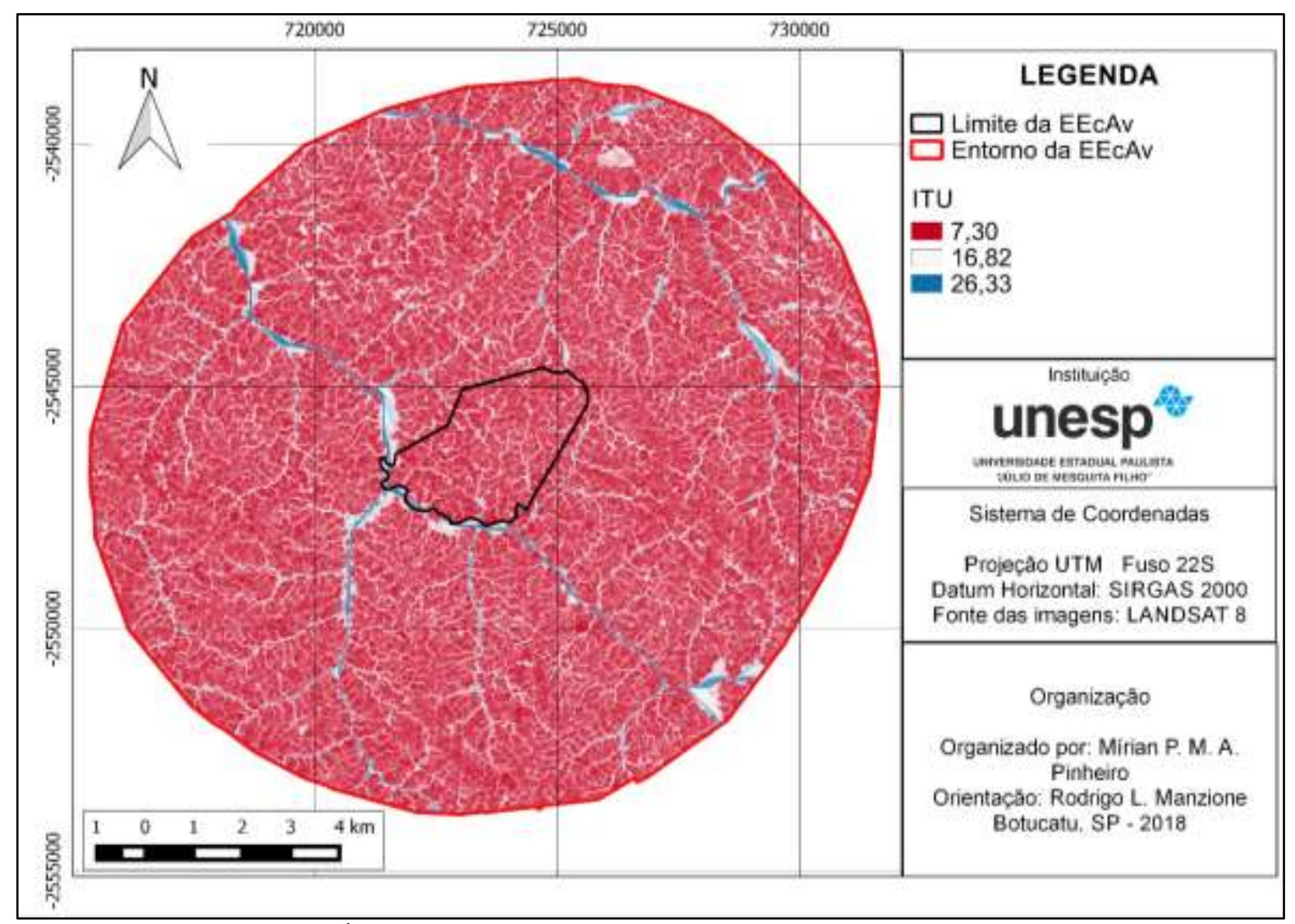

Figura 9. Índice Topográfico de Umidade da EEcAv e seu entorno.

\section{CONCLUSÃO}

Em vista do que foi analisado, é possível concluir que, os mapas de fluxo indicam que grande parte do fluxo subterrâneo da EEcAv está direcionado para os locais com maior umidade, ou seja, os rios Novo e Pardo, tornando a estação

\section{AGRADECIMENTOS}

À CAPES (Coordenação de Aperfeiçoamento de Pessoal de Nível Superior) pela bolsa de doutorado recebida pela primeira autora

Ao FEHIDRO (Fundo Estadual de Recursos Hídricos do Estado de São Paulo)

\section{REFERÊNCIAS BIBLIOGRÁFICAS}

BEVEN, K. J.; KIRKBY, M. J. A physically based, variable contributing area uma contribuinte para a manutenção do fluxo de base dos corpos hídricos superficiais da área. Também é observado nos mapas que a EEcAv está situada entre corredores hídricos, reforçando sua importância ambiental.

- Pojeto 2012-MP-465 pelos recursos financeiros para perfuração e monitoramento dos poços na EEcAv.

Ao IF (Instituto Florestal do Estado de São Paulo) - Projeto COTEC 260108 003.789/2012 pelo suporte de campo.

model of basin hydrology. Hydrologic Science Bulletin, v.24, p.43-69, 1979. 
BUARQUE, D. C.; FAN, F. M.; PAZ, A. R. da; COLLISCHONN, W. Comparação de métodos para definir direções de escoamento a partir de modelos digitais de elevação. Revista Brasileira de Recursos Hídricos, v.14, p.91-103, 2009.

CAPOANE, V.; TIECHER, T.; ALVAREZ, J. W. R. R.; SCHAEFER, G. L.; SANTOS, L. J. C.; SANTOS, D. R. dos. Influência da resolução do modelo digital de elevação na determinação do índice topográfico de umidade e na capacidade de predição dos teores carbono orgânico do solo. Revista Geo UERJ. v. 27, p. 144-155, 2015.

COSTA-CABRAL, M. C.; BURGES, S. J. Digital elevation model networks (DEMON): A model of flow over hillslopes for computation of contributing and dispersal areas. Water Resources Research, v. 30, p. 1681-1692. 1994.

DESMET, P. J. J.; GOVERS, G. Comparison of routing algorithms for digital elevation models and their implications for predicting ephemeral gullies. International Journal of Geographical Information Systems, 10 (3): 311-331. 1996.

EMBRAPA (Empresa Brasileira de
Pesquisa Agropecuária). LANDSAT -
Land Remote Sensing Satellite. 2013.
Disponível
<https://www.cnpm.embrapa.br/projetos/sa
t/conteudo/missao_landsat.html>, acesso
em 4 out 2018.

FREEMAN, T. G. Calculating catchment area with divergente flow based on a regular grid. Computers and Geosciences, v. 17, p. 413- 422. 1991.

GALLANT, J. C.; WILSON, J. P. Primary topographic attributes. In: WILSON, J. P.; GALLANT, J. C. (Ed.). Terrain analysis: principles and applications. New York: John Wiley, p. 51-85. 2000.

GARCIA, M. J. L.; CAMARASA, A. M. Use of geomorphological units to improve drainage network extraction from DEM: Comparision between automated extraction and photointerpretation methods in the Carraixet catchment (Valencia, Spain). International Journal of Applied Earth Observation and Geoinformation, n.3-4, p. 187-194, 1999.

IPT (Instituto de Pesquisas Tecnológicas do Estado de São Paulo). Mapa Geomorfológico do Estado de São Paulo, escala 1:500.000 (Série Monografias n. 5, v.1). São Paulo. 1981.

JENSON, S. K., DOMINGUE, J. O. Extracting topographic structure from digital elevation data for geographic information system analysis.

Photogrammetric Engineering and Remote Sensing, v. 54, p. 1593-1600. 1988.

MANZIONE, R. L. Águas subterrâneas: conceitos e aplicações sob uma visão multidisciplinar. Jundiaí: Paco editorial, 2015. 388p.

MANZIONE, R. L. Incorporação de incertezas associadas à predições de modelos hidrológicos aos instrumentos de gestão em recursos hídricos. Caderno Prudentino de Geografia, v. especial, n. 36, p. 215-227, 2014.

MMA (Ministério do Meio Ambiente). Unidades de Conservação. 2019. Disponível em <https://www.mma.gov.br/areasprotegidas/unidades-de-conservacao/>, acesso em 27 jul 2019.

MELLEK, J. E. Determinação de parâmetros hidrológicos da sub-bacia do Rio Belém usando sistemas de informações geográficas. Especialização (Especialista em geoprocessamento). Universidade Federal do Paraná. Curitiba. 2012.

MITTERMEIER, R. A.; GIL, P. R.; HOFFMANN, M.; PILGRIM, J.; BROOKS, T.; MITTERMEIER, C. G.; LAMOUREX, J.; FONSECA, G. A. B. da. Hotspots Revisitados: As Regiões Biologicamente Mais Ricas e Ameaçadas do Planeta (Mata Atlântica e Cerrado). Conservação Internacional. Brasil. 2005. 
MOORE, I. D.; GESSLER, G. A.; PETERSON, G. A. Soil attribute prediction using terrain analysis. Soil Science Society of America Journal, v.57, p.443-452, 1993.

MYERS, N.; MITTERMEIER, R. A.; MITTERMEIER, C. G.; FONSECA, G. A. B. da; KENT, J. Biodiverity hotspots for conservation priorities. Nature. v. 403, p. 853-858. 2000.

NAVA, A.; MANZIONE, R. L. Resposta de níveis freáticos do sistema Aquífero Bauru (Foramção Adamantina) em função da precipitação e evapotranspiração sob diferentes usos da terra. Águas Subterrêneas, v. 29, p. 191-205, 2015.

O'CALlAGHAN, J. F.; MARK, D. M. The extraction of drainage networks from digital elevation data. Computer Vision, Graphics, and Image Processing, v. 28, p. 323-344. 1984.

OLIVEIRA, A. H.; SILVA, M. A. DA; SILVA, M. L. N.; AVANZI, J. C.; CURI; N.; LIMA, G. C.; PEREIRA, P. H. Caracterização ambiental e predição dos teores de matéria orgânica do solo na SubBacia do Salto, Extrema, MG. Semina: Ciências Agrárias, v. 33, n. 1, p. 143-154, 2012.

OLIVEIRA, L. G. L.; PEREIRA, L. M.; PEREIRA, G.; MORAES, E. C.; MAEDA, E. E. Estudo da variabilidade de índices de vegetação através de imagens do ETM+/LANDSAT 7. In: Simpósio Brasileiro de Sensoriamento Remoto, 13., Florianópolis. Anais... Florianópolis: Instituto Nacional de Pesquisas Espaciais, 2007. p. 5995-6002. 2007a.

OLIVEIRA， S. N. de; CARVALHO JUNIOR; O. A. de; SILVA, T. M. de; GOMES, R. A. T.; MARTINS, E. de S.; GUIMARÃES, R. F.; SILVA, N. C. da. Delimitação automática de bacias de drenagens e análise multivariada de atributos morfométricos usando modelo digital de elevação hidrologicamente corrigido. Revista Brasileira de Geomorfologia, v.8, p. 3-21, 2007b.
PEREIRA, H. M.; LEADLEY, P. W.; PROENÇA, V.; ALKEMADE, R.; SCHARLEMANN, J. P. W.; FERNANDEZ-MANJARRÉS, J. F.; ARAÚJO, M. B.; BALVANERA, P.; BIGGS, R.; CHEUNG, W. W. L.; CHINI, L.; COOPER, H. D.; GILMAN, E. L.; GUÉNETTE, S.; HURTT, G. C.; HUNTINGTON, H. P.; MACE, G. M.; OBERDORFF, T.; REVENGA, C.; RODRIGUES, P.; $\quad$ SCHOLES, R. J.; SUMAILA, U. R.; WALPOLE, M. Scenarios of global diversity in the $21 \mathrm{st}$ century. Science, v. 330, p. 1496-1501, 2010.

QUINN, P.; BEVEN, K.; CHEVALLIER, P.; PLANCHON, O. The prediction of hillslope flow paths for distributed hydrological modeling using digital terrain models. Hydrological Processes, v. 5, p. 59-80. 1991.

RAMOS, V. M., GUIMARÃES, R. F., REDIVO, A. L., CARVALHO JUNIOR, O. A. de, FERNANDES, N. F.; GOMES, R. A. T. Avaliação de metodologias de determinação do cálculo de áreas de contribuição. Revista Brasileira de Geomorfologia, v. 4, p. 41-49. 2003.

ROSA, F. S. Geotecnologias na Geografia Aplicada. Revista do Departamento de Geografia, n. 16, p. 81-90, 2005.

SAFRE, A. L. dos S. Simulação numérica do fluxo das águas subterrâneas na Estação Ecológica de Santa Bárbara/SP. Dissertação (Mestrado em Agronomia) Universidade Estadual Paulista, Faculdade de Ciências Agronômicas. Botucatu. 2018.

SANTAROSA, L. V. Mapeamento de níveis freáticos do Sistema Aquífero Bauru (SAB) em área de proteção ambiental em Águas de Santa Bárbara/SP durante o ano hidrológico 2014/15. Dissertação (Mestrado em Agronomia) - Universidade Estadual Paulista, Faculdade de Ciências Agronômicas. Botucatu. 2016.

SANTAROSA, L. V.; MANZIONE, R. L. Soil variables as auxiliary information in spatial prediction of shallow water table 
levels for estimating recovered water volume. Revista Brasileira de Recursos Hídricos, v. 23, e24, 2018.

SOBRINHO, T. A.; OLIVEIRA, P. T. S.; RODRIGUES, D. B. B.; AYRES, F. M. Delimitação automática de bacias hidrográficas utilizando dados SRTM. Engenharia Agrícola, Jaboticabal, v. 30, p.46-57, 2010.

TARBOTON, D. G. A new method for the determination of flow directions and upslope areas in grid digital elevation models. Water Resources Research, v. 33, p. 309-319. 1997.

THOMAS, C. D.; CAMERON, A.; GREEN, R. E.; BAKKENES, M.; BEAUMONT, L. J.; COLLINGHAM, Y. C.; ERASMUS, B. F.; SIQUEIRA, M. F. de; GRAINGER, A.; HANNAH, L.; HUGHES, L.; HUNTLEY, B.; VAN JAARSVELD, A. S.; MIDGLEY, G. F.; MILES, L.; ORTEGA-HUERTA, M. A.; PETERSON, A. T.; PHILlIPS, O. L.;
WILLIAMS, S. E. Extinction risk from climate change. Nature, v. 427, p. 145148, 2004.

TRIBE, A. Automated recognition of valley lines and drainage networks from grid digital elevation models: A review and a new method. Journal of Hydrology, v.139. p.263-293. 1992.

TUCCI, C. E. M. Hidrologia: ciência e aplicação. 2.ed. Porto Alegre: ABRH/Editora da UFRGS, 1997.

VALERIANO, M.M.; ABDON, M.M. Aplicação de dados SRTM a estudos do Pantanal. Revista Brasileira de Cartografia, Rio de Janeiro, v.59, p.63-71, 2007.

WILSON, J. P.; GALLANT, J. C. Terrain Analysis: Principles and Applications. New York: John Wiley \& Sons, Inc., 2000.

YAMAMOTO, J. L.; LANDIM, P. M. B. Geoestatística: conceitos e aplicações. São Paulo: Oficina de Textos, 2013, 215p. 